\title{
Communication Capacity of Quantum Computation
}

\author{
S. Bose, L. Rallan and V. Vedral \\ Centre for Quantum Computation, Clarendon Laboratory, University of Oxford, Parks Road, Oxford OX1 3PU, England
}

(July 3, 2021)

\begin{abstract}
By considering quantum computation as a communication process, we relate its efficiency to a communication capacity. This formalism allows us to rederive lower bounds on the complexity of search algorithms. It also enables us to link the mixedness of a quantum computer to its efficiency. We discuss the implications of our results for quantum measurement.
\end{abstract}

Any computation, both classical and quantum, is formally identical to a communication in time. At time $t=0$, the programmer sets the computer to accomplish any one of several possible tasks. Each of these tasks can be regarded as embodying a different message. Another programmer can obtain this message by looking at the output of the computer when the computation is finished at time $t=t_{1}$. Recent years have witnessed a surge of interest in both quantum computation [1] 3] and quantum communication [4,5]. Computation based on quantum principles allows for more efficient algorithms for solving certain problems than algorithms based on purely classical principles. Quantum communication, on the other hand, can be used for unconditionally secure secret key distribution [6]. However, till date, these two areas (i.e quantum computation and quantum communication) have developed independently. In this letter we connect the classical capacity of a quantum communication channel [4] with the efficiency of quantum computation. This offers an unifying framework for quantum information processing.

Let us first introduce a few definitions and a communication model of quantum computation. We have two programmers, the sender and the reciever and two registers, the memory $(M)$ register and the computational $(C)$ register. The sender prepares the memory register in a certain quantum state $|i\rangle_{M}$ which encodes the problem to be solved. For example, in the case of factorization [2], this register will store the number to be factored. In case of a database search [3], this register will store the state of the database to be searched. The number $N$ of possible states $|i\rangle_{M}$ will, of course, be limited by the greatest number that the given computer could factor or the largest database it could search. The reciever prepares the computational register in some initial state $\rho_{C}^{0}$. Both the sender and the reciever feed the registers (prepared by them) to the quantum computer. The quantum computer implements the following general transformation on the registers

$$
(|i\rangle\langle i|)_{M} \otimes \rho_{C}^{0} \rightarrow(|i\rangle\langle i|)_{M} \otimes U_{i} \rho_{C} U_{i}^{\dagger}
$$

The resulting state $\rho_{C}(i)=U_{i} \rho_{C}^{0} U_{i}^{\dagger}$ of the computational register contains the answer to the computation and is measured by the reciever. As the quantum computation should work for any $|i\rangle_{M}$, it should also work for any mixture $\sum_{i}^{N} p_{i}(|i\rangle\langle i|)_{M}$, where $p_{i}$ are probabilities. For the sender to use the above computation as a communication protocol, he has to prepare any one of the states $|i\rangle_{M}$ with an apriori probability $p_{i}$. The entire input ensemble is thus $\sum_{i}^{N} p_{i}(|i\rangle\langle i|)_{M} \otimes \rho_{C}^{0}$. Due to the quantum computation, this becomes

$$
\sum_{i}^{N} p_{i}(|i\rangle\langle i|)_{M} \otimes \rho_{C}^{0} \rightarrow \sum_{i}^{N} p_{i}(|i\rangle\langle i|)_{M} \otimes \rho_{C}(i) .
$$

Whereas before the quantum computation, the two registers where completely uncorrelated (mutual information is zero), at the end, the mutual information becomes

$$
\begin{aligned}
I_{M C}: & =S\left(\rho_{M}\right)+S\left(\rho_{C}\right)-S\left(\rho_{M C}\right) \\
& =S\left(\rho_{C}\right)-\sum_{i}^{N} p_{i} S\left(\rho_{C}(i)\right),
\end{aligned}
$$

where $\rho_{M}$ and $\rho_{C}$ are the reduced density operators for the two registers, $\rho_{M C}$ is the density operator of entire $M+C$ system and $S(\rho)=-\operatorname{Tr} \rho \log \rho$ is the von Neumann entropy (for conventional reasons we will use $\log _{2}$ in all calculations). Notice that the value of the mutual information (i.e correlations) is equal to the Holevo bound $H=S\left(\rho_{C}\right)-\sum_{i}^{N} p_{i} S\left(\rho_{C}(i)\right)$ for the classical capacity of a quantum communication channel [1] (Note that $\left.\rho_{C}=\sum_{i}^{N} p_{i} \rho_{C}(i)\right)$. This tells us how much information the reciever can obtain about the choice $|i\rangle_{M}$ made by the sender by measuring the computational register. The maximum value of $H$ is obtained when the states $\rho_{C}(i)$ are pure and orthogonal. Moreover, the sender conveys the maximum information when all the message states have equal apriori probability (which also maximizes the channel capacity). In that case the mutual information (channel capacity) at the end of the computation is $\log N$. Thus the communication capacity $I_{M C}$ (given by Eq.(3)) gives an index of the efficiency of a quantum computation. The target of a quantum computation is to achieve the maximum possible communication capacity consistent with given initial states of the quantum computer. If one breaks down the general unitary transformation $U_{i}$ of a quantum algorithm into several succesive unitary transformations, then the maximum capacity may be achieved only after several steps. In each of the smaller unitary transformations, the mutual information between the $M$ and the $C$ registers (i.e the communication capacity) increases by a certain amount. When its total value reaches 
the maximum possible value consistent with a given initial state of the quantum computer, the computation is regarded as being complete.

We now proceed to illustrate one immediate application of the above formalism. Any general quantum algorithm has to have a certain number of queries into the memory register [7 (this is neccessiated by the fact that the transformation on the computational register has to depend on the problem at hand, encoded in $|i\rangle_{M}$ ). These queries can be considered to be implemented by a black box into which the states of both the memory and the computational registers are fed. The number of such queries needed in a certain quantum algorithm gives the black box complexity of that algorithm [7 9] and is a lower bound on the complexity of the whole algorithm. Recently, Ambainis [9] showed in a very elegant paper that if the memory register was prepared initially in the superposition $\sum_{i}^{N}|i\rangle_{M}$, then, in a search algorithm, $O(\sqrt{N})$ queries would be needed to completely entangle it with the computational register. This gives a lower bound on the number of queries in a search algorithm. In a manner analogous to his, we will calculate the change in mutual information between the memory and the computational registers (from Eq.(3)) in one query step. The number of queries needed to increase the mutual information to $\log N$ (for perfect communication between the sender and the reciever), is then a lower bound on the complexity of the algorithm.

Any search algorithm (whether quantum or classical, irrespective of its explicit form), will have to find a match for the state $|i\rangle_{M}$ of the $M$ register among the states $|j\rangle_{C}$ of the $C$ register and associate a marker to the state that matches (Here, $|j\rangle_{C}$ is a complete orthonormal basis for the $C$ register). The most general way of doing such a query in the quantum case is the black box unitary transformation 9

$$
U_{B}|i\rangle_{M}|j\rangle_{C}=(-1)^{\delta_{i j}}|i\rangle_{M}|j\rangle_{C} .
$$

Any other unitary transformation performing a query matching the states of the $M$ and the $C$ registers, could be constructed from the above type of query. We would like to put a bound on the change of the mutual information in one such black box step. Let the memory states $|i\rangle_{M}$ be available to the sender with equal apriori probability so that the communication capacity is a maximum. His initial ensemble is then $\frac{1}{N} \sum_{i}^{N}(|i\rangle\langle i|)_{M}$. Let the reciever prepare the $C$ register in an initial pure state $\psi^{0}$ (in fact, the power of quantum computation stems from the ability of the reciever to prepare pure state superpositions of form $\left.\frac{1}{N} \sum_{j}^{N}|j\rangle_{C}\right)$. In general, there will be many black box steps on the initial ensemble before perfect correlations between the $M$ and the $C$ registers is set up. Let, after the $k$ th black box step, the state of the system be

$$
\rho^{k}=\frac{1}{N} \sum_{i}^{N}(|i\rangle\langle i|)_{M} \otimes\left(\left|\psi^{k}(i)\right\rangle\left\langle\psi^{k}(i)\right|\right)_{C}
$$

where

$$
\left|\psi^{k}(i)\right\rangle_{C}=\sum_{j} \alpha_{i j}^{k}|j\rangle_{C}
$$

The $(k+1)$ th black box step changes this state to $\rho^{k+1}=\frac{1}{N} \sum_{i}^{N}(|i\rangle\langle i|)_{M} \otimes\left(\left|\psi^{k+1}(i)\right\rangle\left\langle\psi^{k+1}(i)\right|\right)_{C}$ with

$$
\left|\psi^{(k+1)}(i)\right\rangle=\sum_{i, j}^{N} \alpha_{i j}^{k}(-1)^{\delta_{i j}}|i\rangle_{M}|j\rangle_{C} .
$$

Thus we only have to evaluate the difference of mutual information between the $M$ and the $C$ register for the states. This difference of mutual information (when computed from Eq.(3)) can be shown to be the difference $\left|S\left(\rho_{C}^{k+1}\right)-S\left(\rho_{C}^{k}\right)\right|$ 10. This quantity is bounded from the above by [1]

$$
\begin{aligned}
\mid S\left(\rho_{C}^{k+1}\right) & -S\left(\rho_{C}^{k}\right) \mid \leq d_{B}\left(\rho_{C}^{k}, \rho_{C}^{k+1}\right) \log N \\
& -d_{B}\left(\rho_{C}^{k}, \rho_{C}^{k+1}\right) \log d_{B}\left(\rho_{C}^{k}, \rho_{C}^{k+1}\right)
\end{aligned}
$$

where, $d_{B}(\sigma, \rho)=\sqrt{1-F^{2}(\sigma, \rho)}$ is the Bures metric and $F(\sigma, \rho)=\operatorname{Tr} \sqrt{\sqrt{\rho} \sigma \sqrt{\rho}}$ is the fidelity. Using methods similar to Ambainis [9], it can be shown that $F\left(\rho_{C}^{k}, \rho_{C}^{k+1}\right) \geq$ $\frac{N-2}{N}$ from which it follows that

$$
\left|S\left(\rho_{C}^{k+1}\right)-S\left(\rho_{C}^{k}\right)\right| \leq \frac{3}{\sqrt{N}} \log N
$$

This means that at least $O(\sqrt{N})$ steps are needed to produce full correlations (maximum mutual information of value $\log N$ ) between the two registers. This gives the black box lower bound on the complexity of any quantum search algorithm. Of course, we know that there also exists an algorithm achieving this bound due to Grover [3] and this has been proven to be optimal [7, 9, 12].

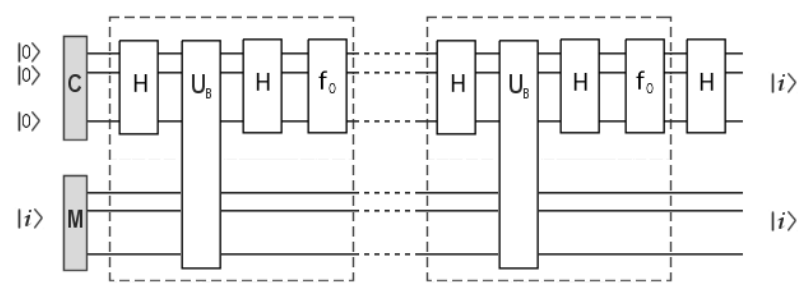

FIG. 1. The figure shows the circuit for Grover's algorithm. $C$ is the computational register and $M$ is the memory register. $U_{B}$ is the black box query transformation, $H$ is a Hadamard transformation on every qubit of the $C$ register and $f_{0}$ is a phase flip in front of the $|00 \ldots 0\rangle_{C}$. The block consisting of $H, U_{B}, H$ and $f_{0}$ is repeated a number of times. 
We now use Grover's algorithm to show how the mutual information varies with time in a quantum search. The general sequence described by Cleve et. al 13 for Grover's algorithm will be used in this letter. The algorithm consists of repeated blocks, each consisting of a Hadamard transform on each qubit of the $C$ register, followed by a $U_{B}$ (our black box transformation), followed by another Hadamard transform on each qubit of the $C$ register and finally a phase flip $f_{0}$ of the the $|00 \ldots 0\rangle_{C}$ state of the $C$ register (See fig.11). This block can then be repeated as many times as is necessary to bring the mutual information to its maximum value of $\log N$, which, as we have shown in Eq.(9) to be $O(\sqrt{N})$. Note that the only transformation correlating the $M$ and $C$ registers is the black box transformation $U_{B}$ and all the other transformations are done only on the $C$ register and therefore do not change the mutual information between the two registers. In fig. 2 we have plotted the variation of mutual information between the $M$ and the $C$ registers (i.e the communication capacity of the quantum computation) with the number of iterations of the block in Grover's algorithm. It is seen that the mutual information oscillates with the number of iterations. Fig.2 is plotted for a four qubit computational register which can search a database of 16 entries. It is seen that the period is roughly 6 , which means that the number of steps needed to achieve maximum mutual information is roughly 3 . This is well above our bound for the minimum number of steps, which is $4 / 3$ in this case.

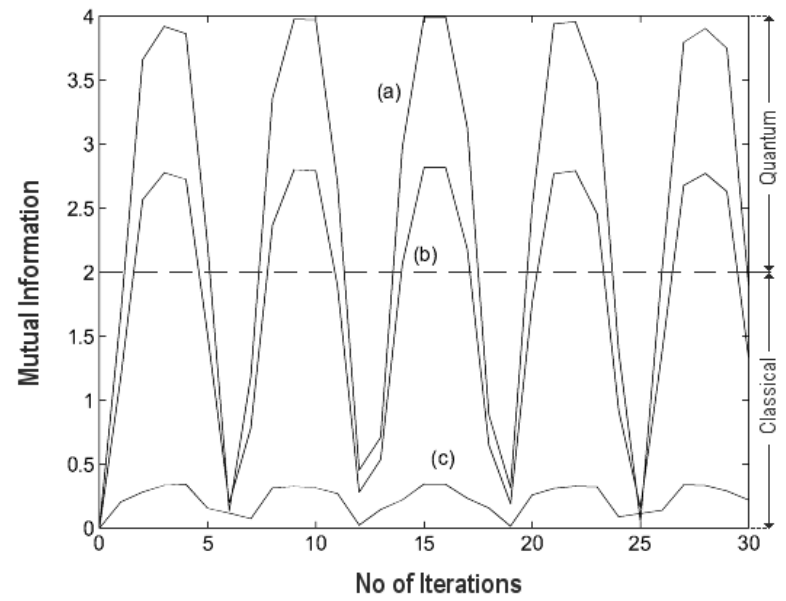

FIG. 2. The figure shows the dependence of the mutual information between the $M$ and the $C$ registers as a function of the number of times the block in Grover's algorithm is iterated for various values of initial mixedness of the $C$ register. Each qubit of the $C$ register is initially in the state $p|0\rangle\langle 0|+(1-p)| 1\rangle\langle 1|$, (a) $p=1$, (b) $p=0.95$ and (c) $p=0.7$. The (a) and (b) computations achieve higher mutual information than classically allowed in the order of root $N$ steps, while (c) does not.

The three graphs (a), (b) and (c) in Fig.2 are for different values of initial mixedness of the $C$ register. We find that the mutual information fails to rise to the maximum value of $\log N$ when the state of the computational register is mixed. Our formalism thus allows us to calculate the performance of a quantum computation as a function of the mixedness (quantified by the von Neumann entropy) of the computational register. We can put a bound on the entropy of the second register after which the quantum search becomes as inefficient as the classical search. If the initial entropy $S\left(\rho_{C}^{0}\right)$ of the $C$ register exceeds $\frac{1}{2} \log N$, then the change in mutual information between the $M$ and the $C$ registers in the course of the entire quantum computation would be at most $\log \sqrt{N}$. This can be achieved by a classical database search in $\sqrt{N}$ steps. So there is no advantage in using quantum evolution when the initial state is too mixed. Note that our condition

$$
S\left(\rho_{C}^{0}\right) \geq \frac{1}{2} \log N
$$

for no quantum speedup in the search algorithm is only a sufficient condition and not a neccessary condition. This is similar to the entropic conditions sufficient to ensure no quantum benefit from teleportation and dense coding [14]. Analogous analysis can be applied to any other algorithm.

Finally, we point out that the states of the $M$ register need not be a mixture, but could be an arbitrary superposition of states $|i\rangle_{M}$ (such a state was used by Ambainis in his argument [9]). All the above arguments still hold in that case, and the $M$ and the $C$ registers become quantum mechanically entangled and not just classically correlated. Thus our analysis implies that any quantum computation is mathematically identical to a measurement process [15]. The system being measured is the $M$ register and the apparatus is the $C$ register of the quantum computer. As the time progresses the apparatus (register $C$ ) becomes more and more correlated (or entangled) to the system (register $M$ ). This means that the states of register $C$ become more and more distinguishable which allows us to extract more information about the $M$ register by measuring the $C$ register. The analysis in the last paragraph, where we showed the limitations on the efficiency of quantum computation imposed by the mixedness of the $C$ register, applies also to the efficiency of a quantum measurement when the apparatus is in a mixed state. Mixedness of an apparatus, to the best of our knowledge, has never been considered in the analysis of quantum measurement. In general practice, any apparatus, however macroscopic, is considered to be in a pure quantum state before the measurement. Our approach highlighting the formal analogy between measurement and computation offers a way to analyse measurement in a much more general context.

L.R. would like to thank Invensys Plc for financial support. 
[1] D. Deutsch, Proc. R. Soc. Lond. A 400, 97 (1985).

[2] P. Shor, SIAM Journal on Computing. 26, 1484 (1997). Also FOCS'94.

[3] L. Grover. A fast quantum mechanical algorithm for database search, Proceedings of the 28th ACM Symposium on Theory of Computing, p. 212, 1996. Also quant$\mathrm{ph} / 9605043$.

[4] A. S. Kholevo, Probl. Peredachi Inf. 9, 177 (1973); Also see A. S. Holevo, IEEE Trans. Info. Th. 44, 269 (1998); B. Schumacher, M. D. Westmoreland, Phys. Rev. A 56, 131 (1997).

[5] C. H. Bennett and G. Brassard, International Conference on Computers, Systems and Signal Processing, Bagalore, India, December 10-12, 1984, pp 175 - 179; C. H. Bennett, and S. Wiesner, Phys. Rev. Lett. 69, 2881 (1992); A. K. Ekert, 1991, Phys. Rev. Lett., 67, 661 (1991); C. H. Bennett, G. Brassard, C. Crepeau, R. Jozsa, A. Peres and W. K. Wooters, Phys. Rev. Lett. 70, 1895 (1993).

[6] D. Deutsch, A. Ekert, R. Jozsa, C. Macchiavello, S. Popescu, and A. Sanpera, Phys. Rev. Lett. 77, 2818 (1996).

[7] C. Bennett, E. Bernstein, G. Brassard and U. Vazirani, SIAM Journal on Computing, 26, 1510 (1997). Also quant-ph/9701001.

[8] R. Beals, H. Buhrman, R. Cleve, M. Mosca and R. de Wolf, Quantum lower bounds by polynomials, Proceedings of FOCS'98, pages 352-361. Also quant-ph/9802049.

[9] A. Ambainis, Quantum lower bounds by quantum arguments, quant-ph/0002066 (2000).

[10] L. Henderson and V. Vedral, Phys. Rev. Lett. 84, 2263 (2000).

[11] M. Fannes, Commun. Math. Phys. 31, 291 (1973); M. Nielsen, Continuity bounds for entanglement, quantph/9908086 (1999).

[12] C. Zalka, Phys. Rev. A 60, 2746 (1999).

[13] R. Cleve, A. Ekert, C. Macchiavello and M. Mosca, Phil. Trans. R. Soc. Lond. A (1997).

[14] S. Bose and V. Vedral, Phys. Rev. A 61, 040101(R), (2000).

[15] J. von Neumann, Mathematical foundations of quantum mechanics, Princeton University Press, Princeton, 1955; H. Everett III, The theory of the universal wave function in The many worlds interpretation of quantum mechanics (B. S. DeWitt and N. Graham eds.), Princeton University Press, Princeton, 1973. 